\title{
ON THE NONSEPARABLE THEORY OF BOREL AND SOUSLIN SETS
}

\author{
BY R. W. HANSELL ${ }^{1}$ \\ Communicated by E. Michael, June 18, 1971
}

Introduction. All spaces considered are assumed to be metrizable. The terminology follows that of [5]. The letter $k$ will denote an infinite cardinal.

The purpose of this note is to introduce the notions of $k$-Souslin and $k$-Borel sets and to announce certain basic results obtained in their study. The results reinforce the feeling that these are the "natural" Borel and Souslin sets to study in nonseparable spaces of weight $k$. The $\aleph_{0}$-Souslin and $\aleph_{0}$-Borel sets are the standard Souslin and Borel sets studied in separable spaces.

The $k$-Borel sets of a space of weight $\leqq k$ can be resolved into "hyperBorel" classes which form an increasing transfinite sequence of type $\omega(k+1)$, the first ordinal of cardinal $k+1$. Moreover, there exist spaces for which these classes are strictly increasing. One important property of $k$-Borel sets which is not a property of Borel sets (in nonseparable spaces) is that a locally $k$-Borel subset of a space of weight $\leqq k$ is $k$-Borel.

Every $k$-Borel subset of a space is a $k$-Souslin subset. On the other hand, the complement of a $k$-Souslin subset may not be $k$-Souslin, so $k$-Souslin subsets need not be $k$-Borel. However, a general form of Souslin's theorem holds: if a set and its complement are $k$-Souslin in a complete space of weight $\leqq k$, then both are $k$-Borel.

Both the $k$-Borel and $k$-Souslin sets are shown to be related to the Baire space of weight $k$ in a way analogous to the relationship which exists between the classical Souslin and Borel sets and the space of irrational numbers.

Proofs and details will appear in [2].

1. $\sigma$-discrete bases and co- $\sigma$-discrete mappings. We recall that a family $\mathscr{B}$ of subsets of a space $X$ is $\sigma$-discrete if $\mathscr{B}=\bigcup_{n=1}^{\infty} \mathscr{B}_{n}$ where each $\mathscr{B}_{n}$ is (relatively) discrete in the sense that for each $x \in B \in \mathscr{B}_{n}$ there exists an open set $O_{X}$ in $X$ such that $O_{X} \cap B^{\prime}=\varnothing$ whenever $B \neq B^{\prime} \in \mathscr{B}_{n}$. (See [8, Lemma 1] for equivalent statements.) A collection $\mathscr{E}$ of subsets of $X$ is said to possess a $\sigma$-discrete base if there exists a $\sigma$-discrete family $\mathscr{B}$ of (not necessarily open) subsets of $X$ such that each set in $\mathscr{E}$ is a union of sets from $\mathscr{B}$.

AMS 1970 subject classifications. Primary 54H05, 04A15; Secondary 54C10, 54E35.

Key words and phrases. $\sigma$-discrete bases, co- $\sigma$-discrete mappings, hyper-Borel set, $k$-Borel set, $k$-Souslin set, Borel classification, metric spaces.

1 This research was supported by a University of Connecticut Faculty Summer Fellowship. 
DEFINITION. We call a mapping $f: X \rightarrow Y$ co- $\sigma$-discrete if the collection $\{f(U) \mid U$ open in $X\}$ has a $\sigma$-discrete base in $Y^{2}$

REMARK. In the nonseparable theory of $(k-)$ Borel and ( $k-)$ Souslin sets, mappings which are merely continuous or Borel measurable rarely preserve structure. This is not the case, however, if the mapping is (in addition) co- $\sigma$-discrete. Note that any mapping with a separable domain is co- $\sigma$ discrete; and so in the separable theory this was always the case.

We list here as propositions some of the more basic properties of co- $\sigma$ discrete mappings.

Proposition 1. If $f: X \rightarrow Y$ is co- $\sigma$-discrete, then so are the following:

(a) $f: X \rightarrow f(X)$.

(b) $f: U \rightarrow Y$, where $U \subset X$ is open.

(c) $f: E \rightarrow Y$, where $E=f^{-1}(M)$ for some $M \subset Y$.

On the other hand, the restriction to a closed subspace need not be co- $\sigma$ discrete.

Proposition 2. If $X$ is separable or $Y$ is $\sigma$-discrete, then every map $f: X \rightarrow Y$ is co- $\sigma$-discrete. Conversely, if $X$ is nonseparable and $Y$ is not $\sigma$-discrete and if $Y$ is complete, then there exists a non-co- $\sigma$-discrete map from $X$ to $Y$.

Proposition 3. Every open map (with a metrizable range) is co- $\sigma$-discrete, and so all projection maps (onto metrizable spaces) are co- $\sigma$-discrete.

Proposition 4. If $Y$ is complete, and $f: X \rightarrow Y$ is 1-1 and takes Borel sets to Borel sets, then $f$ is co- $\sigma$-discrete ( $c f .[3, \S 3.2$, Theorem 5]).

We call the Baire space of weight $k$, and denote by $B(k)[7, \S 2]$, the product space $\prod T_{n}(n=1,2, \ldots)$ where each $T_{n}$ is a discrete space of cardinal $k$. It is well known that $B\left(\aleph_{0}\right)$ is homeomorphic to the space of irrational numbers. We say that a (metric) space $X$ is $\sigma$-locally of weight $<k$ (abbreviated $\sigma$-LW $<k$; see $[8, \S 1]$ and $\left[9\right.$, p. 23]) if $X=\bigcup_{n=1}^{\infty} X_{n}$ where each $x \in X_{n}$ has a neighborhood in $X_{n}$ of weight $<k$. The following theorem extends Theorem V of [5, p. 444] (cf. also [8, Theorem 4]).

THeOREM. Let $X$ be a complete (metric) space, and suppose $f: X \rightarrow Y$ is continuous and co- $\sigma$-discrete. Then either $f(X)$ is $\sigma-L W<k$ or $X$ contains a closed set $C$ homeomorphic to $B(k)$ (or to the Cantor space if $k=\aleph_{0}$ ) such that $f \mid C$ is a homeomorphism.

2. Hyper-Borel sets. The family of Borel sets of a topological space $X$, denoted $B(X)$, is the smallest family of subsets of $X$ which contains (i) the

\footnotetext{
${ }^{2}$ In [3] we called a mapping $f: X \rightarrow Y \sigma$-discrete if the collection $\left\{f^{-1}(V) \mid V\right.$ open in $\left.Y\right\}$ had a $\sigma$-discrete base.
} 
open sets of $X$, (ii) $X-B$ whenever $B \in B(X)$, and (iii) $\bigcup_{n} B_{n}$ whenever each $B_{n} \in B(X)$ and $\left\{B_{n}\right\}$ is a countable family of sets of $X$. We now define the family of hyper-Borel sets of $X$, denoted $H B(X)$, to be the smallest family of subsets of $X$ which contains (i) the open sets of $X$, (ii) $X-B$ whenever $B \in H B(X)$, and (iii) $\bigcup_{t} B_{t}$ whenever each $B_{t} \in H B(X)$ and $\left\{B_{t}\right\}$ is a $\sigma$-discrete family of subsets of $X$.

2.1. Classification of hyper-Borel sets. Given a space $X$, we define hyper-Borel classes $G_{\alpha}=G_{\alpha}(X)$ for each ordinal $\alpha=\lambda+n$ ( $\lambda$ a limit ordinal or 0 and $n=0,1,2, \ldots$ ) as follows :

$G_{0}=$ family of open sets in $X$;

$G_{\lambda+n}=$ all unions (resp. intersections) of countable families from $G_{\lambda+n-1}$ if $n$ is even (resp. odd);

$G_{\lambda}=$ all unions of $\sigma$-discrete families from $\bigcup_{\alpha<\lambda} G_{\alpha}$.

In addition, we define the hyper-Borel classes $F_{\alpha}=F_{\alpha}(X)$ by taking $F_{\alpha}=\left\{X-B \mid B \in G_{\alpha}\right\}$.

THEOREM 1. If $X$ is metrizable, the hyper-Borel classes coincide with the usual Borel classes [5, p. 345] for each countable ordinal $\alpha$. Thus, $B(X)$ $=\bigcup_{\alpha<\omega_{1}} G_{\alpha}(X)=\bigcup_{\alpha<\omega_{1}} F_{\alpha}(X)$.

Theorem 1 is a consequence of the Montgomery theorem [5, p. 358] which, in part, shows that the union of a discrete family of $G_{\alpha}$ (or $F_{\alpha}$ for $\alpha>0)$ sets is again of that class $\left(\alpha<\omega_{1}\right)$.

THEOREM 2. If $X$ is a (metric) space of weight $\leqq k$ and $\omega=\omega(k+1)$ denotes the least ordinal of cardinal $k+1$ (the immediate successor of $k$ ), then $H B(X)=\bigcup_{\alpha<\omega} G_{\alpha}=\bigcup_{\alpha<\omega} F_{\alpha}$.

It is clear that the operations defining the classes $G_{\alpha}$ and $F_{\alpha}$ produce only hyper-Borel sets; that these operations produce nothing new beyond the ordinals of cardinal $k$ is a consequence of the fact that a $\sigma$-discrete family in a space of weight $k$ has at most $k$ members.

2.2. $k$-Borel sets. A second way to classify hyper-Borel sets is to call a set, $B \subset X, k$-Borel if $B$ is hyper-Borel in $X$ of class $\alpha$ for some ordinal $\alpha$ of cardinal $\leqq k$. By Theorem 1 , the $\aleph_{0}$-Borel sets are the ordinary Borel sets.

THEOREM 3. If $B \subset X$ is hyper-Borel and has weight $\leqq k$, then $B$ is $k$-Borel in $X$.

The theorem follows easily from Theorem 2 applied to $\mathrm{Cl}_{X} B$.

2.3. Existence of sets of exact class $\alpha$. If a set is of class $G_{\alpha}$ (or $F_{\alpha}$ ) in a space $X$ but is not of any lower class, then the set is said to be of exact class $\alpha$ in $X$. It is well known [5, p. 371] that the space of irrational numbers $B\left(\aleph_{0}\right)$ contains sets of exact class $\alpha$ for each countable ordinal $\alpha$. This is a special case of the following theorem. 
THEOREM 4. The Baire space $B(k)$ contains sets of exact class $\alpha$ for each ordinal $\alpha$ of cardinal $\leqq k$.

The proof embodies an extension and modification of the techniques used in [1]. Theorem 4 shows that the concept of $k$-Borel sets for $k>\aleph_{0}$ is by no means a superfluous one.

2.4. Absolute $k$-Borel sets. We are mainly concerned with spaces which are absolutely $k$-Borel; i.e., spaces which are $k$-Borel sets in every (metric) space in which they are embedded. Using Lavrentiev's theorem [5, p. 429] one can show that a space $X$ is an absolute $k$-Borel set of class $\alpha$ if, and only if, it is of class $\alpha$ in some complete metric space $(\alpha \geqq 1)$.

THEOREM 5. If $Y$ is an absolute $k$-Borel set of class $\alpha$ and of weight $\leqq k$, there exists a closed subset $A$ of $B(k)$ and a continuous bijection $h: A \rightarrow Y$ such that $f(U)$ is of class $\alpha$ in $Y$ whenever $U$ is open in $A$ (cf. [7, Theorem 4]).

Recent work of A. H. Stone [7], [8], [9] has developed an extensive structure theory for nonseparable absolute Borel sets, which culminates in a complete classification and topological characterization in terms of Borel isomorphism classes. It is the conjecture of this author that most, if not all, of this theory can be extended to the more general class of $k$-Borel sets.

2.5. Locally $k$-Borel sets. A set $E \subset X$ is said to be locally $k$-Borel in $X$ if each $x \in E$ has a neighborhood $U_{X}$ such that $U_{X} \cap E$ is a $k$-Borel subset of $X$.

THEOREM 6. If $E \subset X$ has weight $\leqq k$ and $E$ is locally $k$-Borel in $X$, then $E$ is a $k$-Borel subset of $X$. Furthermore, if $E$ is locally of class $G_{\alpha}\left(F_{\alpha}\right)$ in $X$, then $E$ is of class $G_{\alpha}$ (resp. $F_{\alpha}$ ) in $X$.

3. $k$-Souslin sets. We recall that a set $A \subset X$ is a Souslin [4, p. 203] (analytic [6, p. 207]) subset of $X$ if $A$ can be expressed in the form

$$
A=\bigcup_{t \in B\left(\aleph_{0}\right)} \bigcap_{n=1}^{\infty} F_{t_{1} \ldots t_{n}} \quad\left(t=\left(t_{1}, t_{2}, \ldots\right)\right),
$$

where each $F_{t_{1} \ldots t_{n}}$ is a closed subset of $X$. If now we define $A_{t_{1} \ldots t_{n}}$ to be the union of all the sets $\bigcap_{m=1}^{\infty} F_{s_{1} \ldots s_{m}}$ with $s_{i}=t_{i}$ for $i=1, \ldots, n$, then it can be shown $\left[3\right.$, p. 2] that the system $\left\{A_{t_{1} \ldots t_{n}}\right\}$ has the following properties: $\left(\mathrm{A}_{1}\right) A=\bigcup\left\{A_{t_{1}} \mid t \in B\left(\aleph_{0}\right)\right\}$.

$\left(\mathrm{A}_{2}\right) A_{t_{1} \ldots t_{n}}=\bigcup\left\{A_{s_{1} \ldots s_{n} s_{n+1}} \mid s_{i}=t_{i}, i=1, \ldots, n\right\}$.

$\left(\mathrm{A}_{3}\right)\left\{A_{t_{1} \ldots t_{n}} \mid t \in B\left(\aleph_{0}\right), \quad n=1,2, \ldots\right\}$ has a (countable and hence) $\sigma$-discrete base.

$\left(\mathrm{A}_{4}\right) \bigcap_{n=1}^{\infty} A_{t_{1} \ldots t_{n}}=\bigcap_{n=1}^{\infty} \bar{A}_{t_{1} \ldots t_{n}}$ for each $t \in B\left(\aleph_{0}\right)$ (closure in $X$ ).

Definition. We say $A \subset X$ is a $k$-Souslin subset of $X$ if subsets $A_{t_{1} \ldots t_{n}}$ 
$\subset X$ (not necessarily closed) can be defined so that properties $\left(\mathrm{A}_{1}\right)-\left(\mathrm{A}_{4}\right)$ hold with $B\left(\aleph_{0}\right)$ replaced by $B(k)$.

Note that this definition is more restrictive than the definition of " $k$-analytic" in [7] which requires only that

$$
A=\bigcup_{t \in B(k)} \bigcap_{n=1}^{\infty} A_{t_{1} \ldots t_{n}}=\bigcup_{t \in B(k)} \bigcap_{n=1}^{\infty} \bar{A}_{t_{1} \ldots t_{n}}
$$

Property $\left(A_{0}\right)$ follows from $\left(A_{1}\right),\left(A_{2}\right)$, and $\left(A_{4}\right)$.

3.1. Operations under which $k$-Souslin sets are closed. A family $\left\{A_{d} \mid d \in D\right\}$ of subsets of a space $X$ is said to be $\sigma$-discretely decomposable if, for each $d \in D$, we can write $A_{d}=\bigcup A_{d n}(n=1,2, \ldots)$ so that $\left\{A_{d n} \mid d \in D\right\}$ is discrete in $X$ for each fixed $n$. Such collections were studied extensively in [3], where we showed they arose naturally in the study of Borel measurable mappings.

THeOREM 7. If $A=\bigcup_{t} \bigcap_{n=1}^{\infty} A^{t_{1} \ldots t_{n}}(t \in B(k))$ and each $A^{t_{1} \ldots t_{n}}$ is a $k$-Souslin subset of a space $X$ satisfying

(1) $A^{t_{1} \ldots t_{n}} \cap A=\bigcup\left\{A^{s_{1} \ldots s_{n} s_{n+1}} \cap A \mid s_{i}=t_{i}, i=1, \ldots, n\right\}$ and

(2) $\left\{A^{t_{1} \ldots t_{n}}\right\}$ is $\sigma$-discretely decomposable for fixed $n$, then $A$ is $k$-Souslin in $X$.

Corollary 1. If $A_{1}, A_{2}, \ldots$ is a countable family of $k$-Souslin sets in a space $X$, then $A=\bigcap A_{n}$ and $A^{\prime}=\bigcup A_{n}(n=1,2, \ldots)$ are $k$-Souslin in $X$.

COROLlaRY 2. Each hyper-Borel subset of a space is a $k$-Souslin subset (for every cardinal $k \geqq \aleph_{0}$ ).

Two other important sources of $k$-Souslin sets are the following:

TheOREM 8. A locally $k$-Souslin subset of a space $X$ is $k$-Souslin in $X$.

THEOREM 9. A continuous co- $\sigma$-discrete image of a $k$-Souslin subset of a space of weight $\leqq k$ is a $k$-Souslin subset of the range.

3.2. Absolutely $k$-Souslin sets and co- $\sigma$-discrete mappings. By an absolutely $k$-Souslin set we mean a set which is $k$-Souslin in every (metric) space in which it can be embedded; or, equivalently, if it is $k$-Souslin in some complete metric space (cf. §2.4).

THEOREM 10. Every continuous, co- $\sigma$-discrete image of $B(k)$ is absolutely $k$-Souslin. Conversely, every absolutely $k$-Souslin space of weight $\leqq k$ is a continuous, co- $\sigma$-discrete image of $B(k)$.

3.3 The theorems of Lusin and Souslin. We give here the precise statements of a general form of the Lusin separation theorem and the famous theorem of Souslin. 
Theorem 11 (Generalized Lusin Separation Theorem). If $P$ and $Q$ are two disjoint $k$-Souslin sets in a complete (metric) space $X$ of weight $\leqq k$, then there exists a $k$-Borel set $B$ in $X$ such that $P \subset B$ and $B \cap Q=\varnothing$.

Theorem 12 (Generalized Souslin Theorem). If $A$ and $X-A$ are both $k$-Souslin subsets of a complete (metric) space $X$ of weight $\leqq k$, then both are $k$-Borel in $X$.

One important consequence of the above theorems is the following characterization of absolute $k$-Borel sets.

THEOREM 13. A 1-1, continuous and co- $\sigma$-discrete image of a closed subset of $B(k)$ is absolutely $k$-Borel and of weight $\leqq k$.

The converse has already been noted in Theorem 5 .

\section{REFERENCES}

1. R. Engelking, W. Holsztyński and R. Sikorski, Some examples of Borel sets, Colloq. Math. 15 (1966), 271-274. MR 34 \# 1198.

2. R. W. Hansell, On the non-separable theory of $k$-Borel and $k$-Souslin sets. I, General topology and its applications (submitted).

3. - Borel measurable mappings for non-separable metric spaces, Dissertation, Rochester, N.Y., 1969; also: Trans. Amer. Math. Soc. 161 (1971), 145-169.

4. F. Hausdorff, Set theory, 2nd ed., Chelsea, New York, 1957. MR 19, 111.

5. K. Kuratowski, Topology. Vol. 1, Academic Press, New York; PWN, Warsaw, 1966. MR 36 \# 840.

6. W. Sierpiński, General topology, 2nd ed., Univ. of Toronto Press, Toronto, 1952. MR 14, 394

7. A. H. Stone, Non-separable Borel sets, Rozprawy Mat. 28 (1962), 41 pp. MR 27 \# 2435.

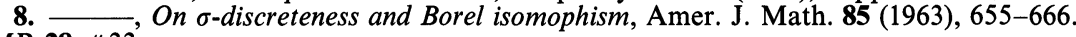
MR 28 \#33.

9. - Proceedings of the Washington State University conference on general topology, Edited and Distributed by Washington Alpha Chapter of Pi Mu Epsilon, 1970. 06268

Department of Mathematics, University of Connecticut, Storrs, Connecticut 